\title{
Design and testing of mechanical heart valve
}

\author{
Meraj Ahmed ${ }^{1}$ Malay K. Das ${ }^{1}$ and Kamal K. Kar ${ }^{1,2 *}$ \\ ${ }^{1}$ Fluid mechanics and thermal sciences, Department of Mechanical Engineering, Indian Institute of \\ Technology Kanpur, Kanpur-208016, India, \\ ${ }^{2}$ Advanced Nanoengineering Materials laboratory, Department of Mechanical Engineering, Indian \\ Institute of Technology Kanpur, Kanpur-208016, India, \\ *Email: kamalkk@iitk.ac.in
}

The human heart acts like a pump, which provides the circulation of blood in the human body. Human heart is divided into four chambers - two atria (left and right) and two ventricles (left and right) - each closed off by a one-way valve. All valves have thin flaps, called leaflets or cusps that open to let the blood flow through them and close to prevent it from flowing backward. Due to heart valve diseases or aging the valve become unable to close and open properly leading to heart failure. Under this situation, diseased valve must be replaced with artificial valve for proper functioning of heart.

The first clinical cardiac valvular prosthesis took place in 1952, when Dr. Charles Hufnagel [1] implanted the first artificial caged ball heart valve in the descending aorta of a patient to treat aortic insufficiency. Since then it has become clear that in order to improve human life the diseased heart valve can be replaced with artificial heart valve. There has been vast improvement in the design of heart valve but still we are far away to achieve the ideal design, which is as durable and as steady as the natural heart valve. Artificial heart valves can be broadly classified into two categories mechanical heart valves (MHV) and bioprosthetic heart valves (BHV). Mechanical heart valves are made of biocompatible material such as titanium, cobalt, pyrolytic carbon, Delrin, Teflon, Dacron, etc. Bioprosthetic heart valves are made of special tissues obtained from animals. BHVs designs have increasingly indicated time dependent (5 to 7 years) structural changes such as calcification and leaflet wear, leading to valve failure [2]. MHVs are made with high strength biocompatible material, which are durable and have long-term functional capability. However, these are subject to thrombus deposition and so patients with implanted mechanical valves need to take long-term anticoagulant therapy. MHVs are generally of three types- caged ball valves, tilting disc valves, bileaflet valves. Caged ball valves are mechanically sound and have built in redundancy in strut design. These have low level of regurgitation in closed phase. But these valves have large height which requires large space for mounting. Tilting disc type valves have better hemodynamic characteristics and lower height than caged ball type. But these have lower level of redundancy in cage strut structure. Lower minor orifice flow leads to tissue over growth and thrombosis. Bileaflet valves provide uniform flow profiles and lower level of structural complications. However, hinge design is prone to thrombosis and valve failure. The performance of mechanical heart valves are related to valve design and structural mechanics. The geometrical features of bileaflet heart valve are shown in Figure 1.

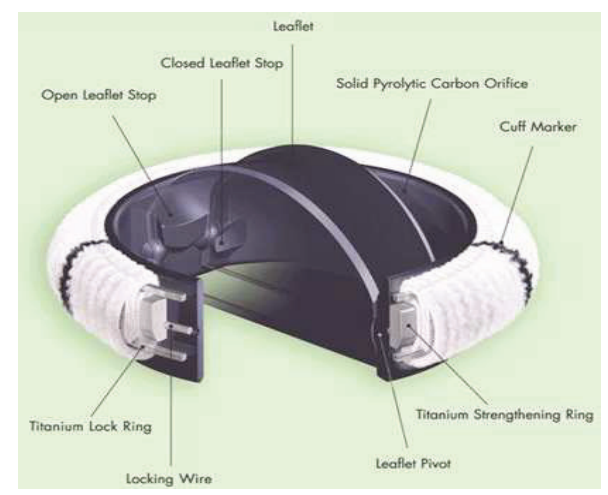

Figure 1: Bileaflet heart valve [3]

\section{References}

1. Hufnagel C A, Harvey W P, Rabil P J, McDermott T F 1954 Surgical correction of aortic insufficiency. Surgery 35: 673-683.

2. Campbell J M 1950 An artificial aortic valve. J. Thorac. Surg. 19: 312-318

3. http://www.pages.drexel.edu 